\title{
FAR-INFRARED EMISSION AND GAS TO DUST RATIO IN DISCS AND CENTRAL REGIONS OF GALAXIES
}

\author{
Y.D. MAYYA \\ INAOE \\ Apdo Postal 52 y 216, 7200 Peubla, Mexico \\ AND \\ T.N. RENGARAJAN \\ Tata Institute of Fundamental Reserach \\ Homi Bhabha Rd, Mumbai 400 005, India
}

We study the radial profiles of radio comtinuum (RC) and far-infrared (FIR) emissions and also the gas to dust ratio for a sample of 22 spiral galaxies. For this, we use the data from IRAS HiRES FIR maps, available VLA $20 \mathrm{~cm}$ maps and radial profile data available on neutral hydrogen $(\mathrm{H})$ and molecular hydrogen $\left(\mathrm{H}_{2}\right.$, derived from velocity integrated $\mathrm{CO}$ intensity). In order to have enough resolution with the 1-1.5 arcminute IRAS beam, the galaxies chosen were large sized and had a $60 \mu \mathrm{m}$ flux density of more than $5 \mathrm{Jy}$. The sample consists of: NGC 628, NGC 2403, NGC 2841, NGC 2903, NGC 3079, NGC 3198, NGC 3627, NGC 3628, NGC 4192, NGC 4254, NGC 4303, NGC 4321, NGC 4501, NGC 4535, NGC 4569, NGC 4656, NGC 4736, NGC 5033, NGC 5055, NGC 6503, NGC 6946 and NGC 7331. For 13 of these galaxies information is also available on metallicity (z) and $\mathrm{V}$ band CCD maps.

From the maps azimuthally averaged radial profiles of RC and FIR $(60 \mu \mathrm{m})$ intensities are obtained using the IRAF package. These profiles are then fitted to a function consisting of a central gaussian component and an exponential disc component. It is found that the two component fit is better than the usually used single exponential fit. In most galaxies, the central gaussian component contains more than half the total emission, the mean fractions in RC and FIR being 0.70 and 0.62 respectively. Further, in agreement with earlier investigations we also find that the gaussian width or the exponential scale height, whichever is dominant is larger for $\mathrm{RC}$ than for FIR. However, the reverse is true for the second exponential component 
in the outer galaxy following the central gaussian. These are found for 20 galaxies in FIR and in only 10 for RC. In 6 galaxies wherein both profiles have outer exponential components, the FIR scale length is larger. This is interpreted as due to FIR emission being sustained in the outer galaxy by dust heated by the interstellar radiation field (ISRF) from old stars while the radio emission falls steeply due to a dearth of young OB stars that give rise to supernovae which power the radio emitting cosmic ray electrons.

From the FIR maps, we derive the dust temperature, $\mathrm{T}_{d}$ using the ratio of fluxes at 60 and $100 \mu \mathrm{m}$ and assuming a $\lambda^{-1}$ dust emissivity dependence. For this purpose we use the dust heating model of Désert et al. (1990) who incorporate three types of grains- PAH, very small grains (VSG) and the normal big grains (BG). The contribution from the ISRF heating is found using the $\mathrm{V}$ band emission after correcting it for extinction given by half the total gas column density for each annulus. We evaluate the the contribution of BG alone after subtracting the other contributions and thus obtain the temperature and optical depth of BG alone. This correction is quite important since VSG contributes significantly to $60 \mu \mathrm{m}$ flux, but very little to the dust mass. The total gas mass is obtained as the sum of $\mathrm{H}$ and $\mathrm{H}_{2}$; the latter is derived from the velocity intgrated intensity of $\mathrm{CO}$ emission assuming a conversion factor $\mathrm{X}$ which has generally been taken as constant (Devereux \& Young, 1990). However, following the finding of Arimoto et al. (1996) we take X to be inversely proportional to metallicity, $z$. We also assume that the gas to dust ratio is independent of $z$. The above assumptions lead to the least gradient in the mean gas to dust profile only a factor of 2 increase towards the centre. Further, in the the outer galaxy (around 0.8 optical radius) its value is 300 , about the same as the Milky Way solar neighbourhood value. It may be noted that while most of the molecular hydrogen and FIR emission is in the inner galaxy, the $\mathrm{H}$ and dust mass continue to be significant in the outer galaxy. Using the $\mathrm{z}$ dependence discussed above and applying VSG correction, we find the global (integrated for the whole galaxy) gas to dust ratio to be 275 while the radial values close to the centre and at the optical radius are 655 and 335 respectively. Thus, we conclude that in spiral galaxies the gas to dust ratio is not very different from that in the Milky Way.

\section{References}

Arimoto, N., Sofue, N., Tsujimoto, T. (1996), PASJ, 48, pp. 275-284

Désert, F.X, Boulanger, F., Puget, J.L. (1990), $A \mathscr{G} A$, 237, pp. 215- 240

Devereux, N.A., Young, J.S. (1990), ApJ, 359, pp.42-56 